International Journal of Pure and Applied Mathematics

Volume 101 No. 1 2015, 87-94

ISSN: 1311-8080 (printed version); ISSN: 1314-3395 (on-line version)

url: http://www.ijpam.eu

doi: http://dx.doi.org/10.12732/ijpam.v101i1.9

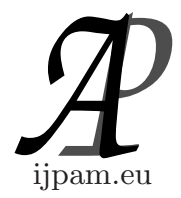

\title{
ON AN INEQUALITY RELATED TO HILBERT'S WITH LAPLACE TRANSFORM
}

\author{
Waleed Abuelela \\ Department of Mathematics \\ Deanery of Academic Services \\ Taibah University \\ University Road, P.O. Box 344, Madina, 41411, KSA \\ Department of Mathematics \\ Faculty of Science \\ Al-Azhar University \\ Nasr City, 11884, Cairo, EGYPT
}

Abstract: Considering different parameters, we obtain new Hilbert-type inequalities involving the Laplace transform. Then we extract from our results some special cases.

AMS Subject Classification: 26D15, 26D07, 26D10, 44A10

Key Words: Hilbert inequality, Hölder inequality, Laplace transform, Gamma function

\section{Introduction}

The well known classical discrete Hilbert-type inequality takes the following form $[1]$ :

If $a_{n}, b_{n} \geq 0,0<\sum_{n=1}^{\infty} a_{n}^{2}<\infty$ and $0<\sum_{n=1}^{\infty} b_{n}^{2}<\infty$, then we have

$$
\sum_{n=1}^{\infty} \sum_{m=1}^{\infty} \frac{a_{m} b_{n}}{m+n} \leq \frac{\pi}{\sin (\pi / p)}\left(\sum_{m=1}^{\infty} a_{m}^{p}\right)^{1 / p}\left(\sum_{n=1}^{\infty} b_{n}^{q}\right)^{1 / q} .
$$

Inequality (1) has the following integral analogous:

Received: February 21, 2015

(c) 2015 Academic Publications, Ltd. url: www.acadpubl.eu 


$$
\int_{0}^{\infty} \int_{0}^{\infty} \frac{f(x) g(y)}{x+y} d x d y \leq \frac{\pi}{\sin (\pi / p)}\left(\int_{0}^{\infty} f^{p}(x) d x\right) \quad\left(\int_{0}^{\infty} g^{q}(x) d x\right)^{1 / q}
$$

unless $f(x) \equiv 0$ or $g(x) \equiv 0$, where $p>1, q=p /(p-1)$. The constant $\pi \operatorname{cosec}(\pi / p)$, in (1) and (2), is the best possible see [1].

Inequalities (1) and (2), which have many generalizations, see for example [2], [3] and references therein, with their improvements have played fundamental roles in the development of many mathematical branches see for instance [2], [4] and references therein.

Recently, some mathematicians have become interested in what so called half-discrete Hilbert-type inequalities see for instance [5] and [6].

In the left-hand sides of this kind of inequalities we have a "mixture" of summations and integrals with a combination between discrete and continuous variables.

For example in [5] we find the following inequality:

If $0<\alpha, 0<r \leq 1, \frac{1}{p}+\frac{1}{q}=1(p \neq 0,1), \lambda_{1}>0, p \lambda_{2} \alpha+(1-p) \lambda_{2} r \leq 1$, $\lambda=\lambda_{1}+\lambda_{2}, a_{n} \geq 0$, and $f(x) \geq 0$ is a real measurable function in $(0, \infty)$, then for $p>1$ :

$$
\begin{aligned}
\sum_{n=1}^{\infty} a_{n} \int_{0}^{\infty} \frac{f(x)}{\left(x^{\alpha}+n^{r}\right)^{\lambda}} d x \leq & \left(\frac{1}{\alpha} \beta\left(\lambda_{1}, \lambda_{2}\right)\right)^{\frac{1}{q}}\left(\frac{1}{r} \beta(\xi, \zeta)\right)^{\frac{1}{p}}\left(\sum_{n=1}^{\infty} n^{q\left(1-\lambda_{2} \alpha\right)-1} a_{n}^{q}\right)^{\frac{1}{q}} \\
& \times\left(\int_{0}^{\infty} x^{p \lambda_{2} \alpha\left(\frac{\alpha}{r}-1\right)+p\left(1-\lambda_{1} \alpha\right)-1} f^{p}(x) d x\right.
\end{aligned}
$$

where $\xi=\lambda_{1}-p \lambda_{2}\left(\frac{\alpha}{r}-1\right)$ and $\zeta=\lambda_{2}+p \lambda_{2}\left(\frac{\alpha}{r}-1\right)$.

In (3), if $r=\alpha=1, p=q=2$, and $\lambda_{1}=\lambda_{2}=\frac{1}{2}$, we obtain the following inequality (which has been proved in [7])

$$
\sum_{n=1}^{\infty} a_{n} \int_{0}^{\infty} \frac{f(x)}{x+n} d x<\pi\left(\sum_{n=1}^{\infty} a_{n}^{2} \int_{0}^{\infty} f^{2}(x)\right)^{1 / 2}
$$

where the constant $\pi$ is the best possible.

In this paper we consider a further generalization of the notion of having a "mixture" of summations and integrals on the left-hand side of the inequality. The upper bound of the inequality under investigation involves Laplace transforms for the functions in the left-hand side of the inequality. 
In the following section we state the main result of this paper of which many special cases can be obtained.

Before proving the main theorem of this paper, Theorem 2.1, let us state and prove the following lemma:

Lemma 1.1. (see [8]) For $n \in \mathcal{N}$, if $\alpha>-\frac{1}{n}, f(x)$ and $g(x)$ are nonnegative measurable functions on the interval $(0, \infty)$, then

$$
\int_{0}^{\infty} \int_{0}^{\infty} \frac{f(x) g(y)}{(x+y)^{\alpha n+1}} d x d y=\frac{1}{\Gamma(\alpha n+1)} \int_{0}^{\infty} s^{\alpha n} F(s) G(s) d s,
$$

where $F(s)$ and $G(s)$ are Laplace transforms of $f(x)$ and $g(x)$ respectively, and $\Gamma(z)$ is the gamma function.

Proof. Using the Fubini's theorem, we have

$$
\begin{aligned}
\int_{0}^{\infty} s^{\alpha n} F(s) G(s) d s & =\int_{0}^{\infty} s^{\alpha n} d s \int_{0}^{\infty} e^{-s x} f(x) d s \int_{0}^{\infty} e^{-s y} g(y) d s \\
& =\int_{0}^{\infty} f(x) d x \int_{0}^{\infty} g(y) d y \int_{0}^{\infty} s^{\alpha n} e^{-s(x+y)} d s \\
& =\int_{0}^{\infty} f(x) d x \int_{0}^{\infty} g(y) d y \int_{0}^{\infty} \frac{t^{\alpha n} e^{-t}}{(x+y)^{\alpha n+1}} d t \\
& =\Gamma(\alpha n+1) \int_{0}^{\infty} \int_{0}^{\infty} \frac{f(x) g(y)}{(x+y)^{\alpha n+1}} d x d y .
\end{aligned}
$$

This completes the proof.

\section{Main Results}

In this section we state and discuss our main theorem together with its special cases.

Theorem 2.1. Suppose that $\alpha>-\frac{1}{n}, \frac{1}{p}+\frac{1}{q}=1, a_{n} \geq 0$, and $f(x), g(x) \geq 0$ are real measurable functions on $(0, \infty)$. Then for $p>1$, the 
following inequalities hold:

$$
\begin{aligned}
& J:=\left(\sum_{n=1}^{\infty} \frac{n^{p \alpha-1}}{(\Gamma(\alpha n+1))^{p}}\left[\int_{0}^{\infty} s^{\alpha n} F(s) G(s) d s\right)^{p}\right. \\
& \leq\left(\sum_{n=1}^{\infty} \frac{n^{p \alpha-1}}{(\Gamma(\alpha n+1))^{p}}\left(\int_{0}^{\infty} s^{p\left(\frac{\alpha n}{2}+\frac{1-\alpha}{q}\right)} F^{p}(s) d s\right)\left(\int_{0}^{\infty} s^{\frac{\alpha n q}{2}+\alpha-1} G^{q}(s) d s\right)^{\frac{p}{q}}\right)^{\frac{1}{p}} \\
& I:=\sum_{n=1}^{\infty} a_{n} \int_{0}^{\infty} \int_{0}^{\infty} \frac{f(x) g(y)}{(x+y)^{\alpha n+1}} d x d y \\
& \leq\left(\sum_{n=1}^{\infty} \frac{n^{p \alpha-1}}{(\Gamma(\alpha n+1))^{p}}\left(\int_{0}^{\infty} s^{p\left(\frac{\alpha n}{2}+\frac{1-\alpha}{q}\right)} F^{p}(s) d s\right)\left(\int_{0}^{\infty} s^{\frac{\alpha n q}{2}+\alpha-1} G^{q}(s) d s\right)^{\frac{p}{q}}\right)^{\frac{1}{p}} \\
& \times\left(\sum_{n=1}^{\infty} n^{q(1-\alpha)-1} a_{n}^{q}\right)^{\frac{1}{q}}
\end{aligned}
$$

where $F(s)$ and $G(s)$ are Laplace transforms for the functions $f(x)$ and $g(x)$ respectively.

Proof. Using Hölder's inequality produces

$$
\begin{aligned}
{\left[\int_{0}^{\infty} s^{\alpha n} F(s) G(s) d s=\right.} & {\left[\int_{0}^{\infty} s^{\frac{\alpha n}{2}} \frac{s^{(1-\alpha) / q} \Gamma(\alpha n+1)}{n^{(1-\alpha) / p}}\right.} \\
& \left.F(s) s^{\frac{\alpha n}{2}} \frac{n^{(1-\alpha) / p}}{\Gamma(\alpha n+1) s^{(1-\alpha) / q}} G(s) d s\right]^{p} \\
\leq & \int_{0}^{\infty} s^{p\left(\frac{\alpha n}{2}+\frac{1-\alpha}{q}\right)} \frac{(\Gamma(\alpha n+1))^{p}}{n^{(1-\alpha)}} F^{p}(s) d s \\
& \times\left[\int_{0}^{\infty} s^{\frac{\alpha n q}{2}-(1-\alpha)} \frac{n^{q(1-\alpha) / p}}{(\Gamma(\alpha n+1))^{q}} G^{q}(s) d s\right]^{\frac{p}{q}}
\end{aligned}
$$




$$
=\left[\int_{0}^{\infty} s^{p\left(\frac{\alpha n}{2}+\frac{1-\alpha}{q}\right)} F^{p}(s) d s\right]\left[\int_{0}^{\infty} s^{\frac{\alpha n q}{2}+\alpha-1} G^{q}(s) d s\right]^{\frac{p}{q}} .
$$

Using Lebesgue term-by-term integration theorem (see [9]) and (9), then inequality (6) can be written as follows

$$
J \leq\left(\sum_{n=1}^{\infty} \frac{n^{p \alpha-1}}{(\Gamma(\alpha n+1))^{p}} \int_{0}^{\infty} s^{p\left(\frac{\alpha n}{2}+\frac{1-\alpha}{q}\right)} F^{p}(s) d s \times\left[\int_{0}^{\infty} s^{\frac{\alpha n q}{2}+\alpha-1} G^{q}(s) d s\right)^{\frac{p}{q}}\right)^{\frac{1}{p}}
$$

This completes the proof of (6). To prove (7), applying Lemma 1.1 then by Hölder's inequality and inequality (6) we obtain

$$
\begin{aligned}
& I:=\sum_{n=1}^{\infty} a_{n} \int_{0}^{\infty} \int_{0}^{\infty} \frac{f(x) g(y)}{(x+y)^{\alpha n+1}} d x d y \\
& =\sum_{n=1}^{\infty}\left(n^{\frac{1}{p}-\alpha} a_{n}\right)\left(\frac{n^{\alpha-\frac{1}{p}}}{\Gamma(\alpha n+1)} \int_{0}^{\infty} s^{\alpha n} F(s) G(s) d s\right) \\
& \leq\left[\sum_{n=1}^{\infty} \frac{n^{\left(\alpha-\frac{1}{p}\right) p}}{(\Gamma(\alpha n+1))^{p}}\left(\int_{0}^{\infty} s^{\alpha n} F(s) G(s) d s\right) \mid\left[\sum_{n=1}^{p} n^{\frac{1}{p}}\left[\frac{1}{p}-\alpha\right) q a_{n}^{q}\right]^{\frac{1}{q}}\right. \\
& =J\left[\sum_{n=1}^{\infty} n\left(\frac{1}{p}-\alpha\right) q a_{n}^{q}\right]^{\frac{1}{q}} \\
& \leq\left(\sum_{n=1}^{\infty} \frac{n^{p \alpha-1}}{(\Gamma(\alpha n+1))^{p}}\left(\int_{0}^{\infty} s^{p\left(\frac{\alpha n}{2}+\frac{1-\alpha}{q}\right)} F^{p}(s) d s\right)\left(\int_{0}^{\infty} s^{\frac{\alpha n q}{2}+\alpha-1} G^{q}(s) d s\right)\right. \\
& \times\left(\sum_{n=1}^{\infty} n^{q(1-\alpha)-1} a_{n}^{q}\right)^{\frac{1}{q}}
\end{aligned}
$$

This completes the proof.

Some special cases of Theorem 2.1 are as follows: 
Case 1. If $\alpha=\frac{1}{p}$, then inequality (7) takes the form:

$$
\begin{aligned}
& \sum_{n=1}^{\infty} a_{n} \int_{0}^{\infty} \int_{0}^{\infty} \frac{f(x) g(y)}{(x+y)^{\frac{n}{p}+1}} d x d y \leq \\
& \left(\sum_{n=1}^{\infty} \frac{1}{\left(\frac{n}{p} \Gamma\left(\frac{n}{p}\right)\right)^{p}} \int_{0}^{\infty} s^{\frac{n}{2}+\frac{p}{q^{2}}} F^{p}(s) d s\left(\int_{0}^{\infty} s^{\frac{n q-2 p}{2 p q}} G^{q}(s) d s \mid\right)_{n=1}^{\frac{1}{p}}\left(\sum_{n}^{\infty} a^{q}\right)^{\frac{1}{q}} .\right.
\end{aligned}
$$

Using the elementary inequality (see [[10], p. 30])

$$
x^{\frac{1}{p}} y^{\frac{1}{q}} \leq \frac{x}{p}+\frac{y}{q}
$$

where $x>0 y>0$ and $\frac{1}{p}+\frac{1}{q}=1$ with $p>1$, then inequality (10) gives the estimate

$$
\begin{aligned}
& \sum_{n=1}^{\infty} a_{n} \int_{0}^{\infty} \int_{0}^{\infty} \frac{f(x) g(y)}{(x+y)^{\frac{n}{p}+1}} d x d y \\
& \leq \frac{1}{p}\left(\sum_{n=1}^{\infty} \frac{1}{\left(\frac{n}{p} \Gamma\left(\frac{n}{p}\right)\right)^{p}} \int_{0}^{\infty} s^{\frac{n}{2}+\frac{p}{q^{2}}} F^{p}(s) d s\left(\int_{0}^{\infty} s^{\frac{n q-2 p}{2 p q}} G^{q}(s) d s \mid\right)^{\frac{p}{q}}\right. \\
& +\frac{1}{q}\left(\sum_{n=1}^{\infty} a_{n}^{q}\right) .
\end{aligned}
$$

However, when $p=q=2$ inequality (10) becomes

$$
\begin{aligned}
& \sum_{n=1}^{\infty} a_{n} \int_{0}^{\infty} \int_{0}^{\infty} \frac{f(x) g(y)}{(x+y)^{\frac{n}{2}+1}} d x d y \\
& \leq\left(\sum_{n=1}^{\infty} \frac{1}{\left(\frac{n}{2} \Gamma\left(\frac{n}{2}\right)\right)^{2}} \int_{0}^{\infty} s^{\frac{n}{2}+\frac{1}{2}} F^{2}(s) d s \int_{0}^{\infty} s^{\frac{n}{4}-\frac{1}{2}} G^{2}(s) d s\right. \\
&
\end{aligned}
$$

Case 2. If $\alpha=1$ and $p=q=2$, then inequality (7) yields: 


$$
\begin{aligned}
\sum_{n=1}^{\infty} a_{n} \int_{0}^{\infty} \int_{0}^{\infty} \frac{f(x) g(y)}{(x+y)^{n+1}} d x d y & \\
& \leq\left(\sum_{n=1}^{\infty} \frac{1}{n(\Gamma(n))^{2}} \int_{0}^{\infty} s^{n} F^{2}(s) d s \int_{0}^{\infty} s^{n} G^{2}(s) d s \mid\left(\sum_{n=1}^{\infty} \frac{a_{n}^{2}}{n}\right)^{\frac{1}{2}} .\right.
\end{aligned}
$$

Case 3. If $\alpha=0$ and $p=q=2$, then inequality (7) produces:

$$
\begin{aligned}
\sum_{n=1}^{\infty} a_{n} \int_{0}^{\infty} \int_{0}^{\infty} \frac{f(x) g(y)}{x+y} d x d y & \\
& \leq\left(\sum_{n=1}^{\infty} \frac{1}{n}\right)^{\frac{1}{2}}\left(\int_{0}^{\infty} s F^{2}(s) d s\right)\left(\int_{0}^{\frac{1}{2}} \frac{1}{s} G^{2}(s) d s\right)\left(\sum_{n=1}^{\infty} n a_{n}^{2}\right)^{\frac{1}{2}} .
\end{aligned}
$$

\section{References}

[1] G. H. Hardy, J. E. Littlewood and G. Polya, Inequalities, Cambridge University Press (1934).

[2] Z. Chang-Jian and C. Wing-Sum, On Hilbert type inequality, J. of Inequalities and Applications 2012:145 (2012).

[3] A. Waleed, Short note on Hilbert's inequality, J. of the Egyptian Mathematical Society 22 (2014), 174-176.

[4] Q. Huang and Y. Bicheng, A multiple Hilbert-type integral inequality with a non-homogeneous kernel, J. of Inequalities and Applications 2013:245 (2013), 248-265.

[5] A. Waleed, A generalization of a half-discrete Hilbert's inequality, submitted to J. of the Egyptian Mathematical Society, expected to appear on (2015), .

[6] Q. Huang and Y. Bicheng, On a more accurate half-discrete Hilbert's inequality, J. of Inequalities and Applications 106 (2012).

[7] Y. Bicheng, A half-discrete Hilbert's inequality, J. Guangdong Univ. Educ. 31(3) (2011), 1-8. 
[8] T. C. Peachey, Some integral inequalities related to Hilbert's, J. of Inequalities in Pure and Appl. Math 4 (2003).

[9] David M. Bressoud, A radical approach to Lebesgue's theory of integration, Cambridge University Press First (2008).

[10] D. S. Mitrinovic, Analytic Inequalities, Springer-Verlag, Berlin/New York, (1970). 